\title{
Effects of oocyte quality and semen donor on the efficiency of in vitro embryo production in cattle*
}

\author{
L. Kątska-Książkiewicz ${ }^{1}$, J. Opiela ${ }^{2}$ and B. Ryńska² \\ ${ }^{1}$ Rzeszow University, Department of Bioengineering, \\ Branch Campus of the Faculty of Biotechnology \\ 36-100 Kolbuszowa, Poland \\ ${ }^{2}$ National Research Institute of Animal Production, \\ Department of Biotechnology of Animal Reproduction \\ 32-083 Balice, Poland
}

(Received 4 March 2008; revised version 9 December 2008; accepted 20 March 2009)

\begin{abstract}
The aim of the study was to determine whether immature bovine oocytes selected by morphology and BCB test (brilliant cresyl blue) and used for in vitro embryo production would increase the blastocyst rate when inseminated with sperm derived from bulls of reduced in vitro fertilizability. To relate oocyte quality and bull effect to blastocyst quality, morphology, ability for hatching and caspase-3 activity (marker of apoptosis) were evaluated.

Both, oocytes originating from $\mathrm{BCB}+$ and from Control COCs exhibited higher cleavage and blastocyst rates $(\mathrm{P}<0.001)$ compared with $\mathrm{BCB}$ - oocytes. Significant differences in cleavage and blastocyst rates between the two bulls were observed. The highest blastocyst rate was noticed for $\mathrm{BCB}+$ oocytes, regardless the bull. Comparison of the caspase- 3 activity shows a significant overbalance of blastocysts with high caspase- 3 activity, regardless the stage of blastocyst development and their origin. Thus, using excellent quality oocytes and sperm with reduced in vitro fertilizability it was possible to some extent to overcome bull effect on embryo production.
\end{abstract}

KEY WORDS: cattle, COCs morphology, BCB test, sperm, caspase-3 activity

\section{INTRODUCTION}

Development of immature bovine oocytes to the blastocyst stage following in vitro maturation (IVM), fertilization (IVF) and culture (IVC) generally occurs in

\footnotetext{
* Supported by the Statutory Activity of the National Research Institute of Animal Production

${ }^{1}$ Corresponding author: e-mail: 1katska@izoo.krakow.pl
} 
approximately $30-40 \%$ of the cases. Unfortunately, none of the attempts leading to improve culture conditions did not significantly increase the efficiency (Lonergan et al., 2003; Hasler, 2006; Pavlok et al., 2006; Sirard et al., 2006; Bols et al., 2007). However, such efficiency has allowed for the field application of in vitro embryo production (IVP) technology in cattle (Hasler, 2006).

It has been previously shown that IVP in cattle and other farm animals is affected by several factors, and the quality of IVP embryos is inferior to those produced in vivo (Rizos et al., 2002). There is now evidence suggesting that the intrinsic quality of the oocyte is the key factor determining the proportion of oocytes developing to the blastocyst stage (Rizos et al., 2002; Lonergan et al., 2003; Vassena et al., 2003; Hasler, 2006; Sirard et al., 2006).

As it has been already demonstrated, in vitro developmental competence of oocytes is highly related to follicular diameter; oocytes derived from large follicles are more competent than those from small follicles (Machatkova et al., 2004). In addition, oocytes matured in vivo are more competent than those matured in vitro (Blondin et al., 2002; Rizos et al., 2002). Moreover, it has been shown that developmental competence may differ significantly among oocytes with the same COC morphology and follicle size and the same extent of cumulus expansion (Han et al., 2006). These observations suggest that the developmental potential of an oocyte is determined by multifactorial interactions (Han et al., 2006).

Many attempts have been made to enhance the developmental competence of oocytes by improving the protocol of in vitro embryo production. The main task is to select the superior oocytes for IVM. Immature oocytes are routinely selected for IVM by visual assessment of morphological features. Although morphological criteria provide a reasonable means of identifying oocyte quality and suitability for IVM, there is evidence that morphological criteria are not sufficient for selection of oocytes of superior developmental potential (Aguilar et al., 2002; Han et al., 2006; Bols et al., 2007). It was clearly demonstrated that immature oocytes selected by similar morphological features differ in their developmental potential. Such a conclusion can be based on observation, that up to $60 \%$ of IVM/IVF oocytes fail to reach the blastocyst stage in vitro (Rizos et al., 2002; Lonergan et al., 2003; Alm et al., 2005; Kątska-Książkiewicz et al., 2005, 2006; Hasler, 2006; Sirard et al., 2006). Therefore, non-invasive markers of oocyte quality that allow for accurate and fast selection prior to IVM have become of prime importance. It has been demonstrated that brilliant cresyl blue (BCB) can be used for the selection of competent oocytes in pig, goat and cattle (Rodriguez-González et al., 2003; Pujol et al., 2004; Alm et al., 2005; Wongsrikeao et al., 2006; Bhojwani et al., 2007; Kątska-Książkiewicz et al., 2007). Although it was previously shown that $\mathrm{BCB}$ staining is a useful tool for selecting more competent oocytes, the test is still not routinely included in laboratory selection protocols. The BCB test allows the determination of the activity of glucose-6-phosphate dehydrogenase (G6PDH), an 
enzyme synthesized in growing oocytes but inactive in oocytes that have finished their growth phase. Brilliant cresyl blue is a dye that can be broken down by G6PDH (Tian et al., 1998). Oocytes during the growth phase and those that have completed their growth will therefore differ in G6PDH level. Oocytes lacking G6PDH activity stain blue.

The success of IVP is also affected by sperm susceptibility to capacitation. It has been shown that in cattle, as in other farm animals, the male effect on IVP leads to high variability in fertilization rates and the efficiency of embryo development (Chamberland et al., 2001; Vassena et al., 2003; Palma and Sinowatz, 2004; Kątska-Książkiewicz et al., 1996, 2005, 2006). The question arises whether it would be possible to overcome the bull effect and increase efficiency of blastocysts production by using selected, competent oocytes for IVF. Since no comparison between interactions of frozen-thawed bull sperm on efficiency of embryo production using oocytes selected by BCB test has been performed, we decided to compare IVP outcomes using frozen-thawed sperm of two bulls that in our previous experiments showed reduced blastocyst rates in comparison to other males (Kątska-Książkiewicz et al., 2005). It was hypothesized that using semen of such bulls IVP outcomes may be improved by introducing oocytes selected by the BCB staining.

Thus, the aim of the present study was to determine whether using immature oocytes selected by COC morphology and brilliant cresyl blue staining would be helpful in increasing IVP efficiency when frozen-thawed semen of bulls with reduced in vitro fertilizability was used. Additionally, to relate two selection criteria of immature oocytes (morphology and $\mathrm{BCB}$ staining) as well as bull effect to quality of blastocysts developing in vitro, blastocysts were evaluated by their morphology, ability for hatching and caspase-3 activity which is an accepted marker of apoptosis.

\section{MATERIAL AND METHODS}

Unless otherwise indicated, all plastic-ware (e.g., culture vessels, dishes and tubes) used in our experiments was obtained from Nunc (Wiesbaden, Germany), while all chemicals and media were purchased from Sigma (Poznań, Poland).

\section{Oocyte recovery and selection}

Ovaries from slaughtered cows and heifers were rinsed 3 times in warm $\left(>30^{\circ} \mathrm{C}\right)$ PBS supplemented with $0.075 \mathrm{~g} / 1$ kanamycine (Kątska-Książkiewicz et al., 2005). Cumulus-oocyte complexes (COCs) were freed from ovaries following the isolation and subsequent rupture of vesicular follicles $2-8 \mathrm{~mm}$ in diameter 
in TCM 199 holding medium (Earle's salt with 25 mM HEPES containing 10\% foetal calf serum (FCS)). COCs with a homogenous cytoplasm were pooled into fresh manipulation medium and held at $38^{\circ} \mathrm{C}$ until subjected to BCB staining. Degenerate oocytes and those without cumulus cells were discarded.

\section{$B C B$ staining}

Compact COCs were washed in warm Dulbecco's PBS supplemented with $0.4 \%$ BSA (A-7888), and then placed in $26 \mu \mathrm{M}$ of BCB (B-5388) diluted in Dulbecco's PBS for $60 \mathrm{~min}$ at $38^{\circ} \mathrm{C}$ in a humidified air atmosphere (RodriguezGonzález et al., 2003; Wongsrikeao et al., 2006). Following BCB exposure, the COCs were washed twice in a warm solution of PBS with $0.4 \%$ BSA. After washing, the COCs were examined under a stereomicroscope and divided into two groups according to their cytoplasm colouration: $\mathrm{BCB}+$ (oocytes with blue ooplasm) and BCB- (colourless). After classification, COCs were washed twice in maturation medium and placed in culture.

The COCs of the control group were evaluated only morphologically and then washed twice in maturation medium and immediately placed in culture. We decided to place control oocytes in culture without holding for $60 \mathrm{~min}$ prior to IVM. As it has been shown by Alm et al. (2005), no significant differences were observed in chromatin configuration in bovine oocytes at the time of recovery (control), and after $90 \mathrm{~min}$ of incubation in medium (holding), nor after $24 \mathrm{~h}$ of in vitro maturation. There was no significant difference in the proportion of MII oocytes between the control and holding groups (Alm et al., 2005). Moreover, no differences were observed in cleavage and blastocysts rates between the control and holding groups (Alm et al., 2005).

\section{In vitro maturation}

The procedure used in our previous experiments was applied (KattskaKsiążkiewicz et al., 2005, 2006). Briefly, COCs were matured in modified TCM 199 containing Earle's salt buffered with sodium bicarbonate, $\mathrm{pH} 7.4$ supplemented with $20 \%$ oestrus cow serum (ECS, heat inactivated) and an additional 3 to 5 $\times 10^{6}$ granulosa cells $/ \mathrm{ml}$. COCs were cultured for 22 to $23 \mathrm{~h}$ at $38.5^{\circ} \mathrm{C}$ under $5 \%$ $\mathrm{CO}_{2}$ at maximum humidity.

\section{In vitro fertilization}

After IVM, oocytes were fertilized in vitro using frozen-thawed sperm from two donors (bulls P and R). Sperm samples were obtained from Polish Animal Breeding stations. Samples of frozen semen of these bulls contained at least $50 \%$ progressively motile spermatozoa after thawing. Both frozen-thawed semen samples 
of these bulls showed similar parameters concerning concentration, type of motility, percentage of motile spermatozoa, quality of chromatin structure evaluated by Sperm Chromatin Structure Assay (SCSA test; Kątska-Książkiewicz et al., 2005) and survival of spermatozoa following thawing. In our previous experiments these semen samples were evaluated as less suitable for IVF because of the reduced blastocyst rates (Kątska-Książkiewicz et al., 2005).

Straws of semen were thawed in a water bath at $37^{\circ} \mathrm{C}$ for $1 \mathrm{~min}$ and kept in the water bath for an additional $5 \mathrm{~min}$. The capacitation procedure was previously described (Kątska-Książkiewicz et al., 2005, 2006). Briefly, spermatozoa were separated on a discontinuous Percoll (Pharmacia, Uppsala, Sweden) density gradient ( $1 \mathrm{ml} \mathrm{45 \%} \mathrm{Percoll} \mathrm{over} 1 \mathrm{ml} \mathrm{90 \%} \mathrm{Percoll)} \mathrm{by} \mathrm{centrifugation} \mathrm{for} 30$ min at $300 \mathrm{~g}$ at room temperature, washed in calcium ion-free TALP medium and pelleted by centrifugation at $100 \mathrm{~g}$ for $10 \mathrm{~min}$. Concentration of spermatozoa was estimated by haemocytometer and sperm samples were diluted to approximately 3 to $5 \times 10^{7}$ sperm $/ \mathrm{ml}$ in the calcium ion-free TALP medium. This suspension was added to the fertilization drops containing $10 \mu \mathrm{g} / \mathrm{ml}$ heparin and mixture of penicillamine $(20 \mu \mathrm{M})$, hypotaurine $(10 \mu \mathrm{M})$ and epinephrine $(1 \mu \mathrm{M})$ at a concentration of $1-2 \times$ $10^{6}$ spermatozoa $/ \mathrm{ml}$ of medium.

Mature COCs were washed and partially released from the expanded cumulus cells before being transferred in groups into $40 \mu 1$ of TALP-IVF medium. Gametes were co-incubated for 20 to $22 \mathrm{~h}$ at $38.5^{\circ} \mathrm{C}$ under $5 \% \mathrm{CO}_{2}$ in air (KattskaKsiążkiewicz et al., 2005, 2006).

\section{In vitro embryo culture}

After 20 to $22 \mathrm{~h}$ of gamete co-incubation, oocytes were washed 3 times with a holding medium to remove corona cells and attached spermatozoa, then transferred into $50 \mu \mathrm{l}$ drops of $\mathrm{B}_{2}$ medium (C.C.D., Paris, France) under mineral oil for 20 to $24 \mathrm{~h}$ (40 to $44 \mathrm{~h}$ post insemination). Next, the cleavage rate was recorded. Uncleaved ova were discarded and embryos were placed in co-culture with Vero cells in $50 \mu 1$ drops of $\mathrm{B}_{2}$ medium supplemented with $2.5 \%$ FCS, under mineral oil. Medium in culture drops was partially changed (20 $\mu \mathrm{l})$ at intervals of $48 \mathrm{~h}$. Embryos were maintained in co-culture for 7 to 8 days. At the end of the culture period, the total blastocyst rate was recorded.

\section{Preparation of Vero cells}

The frozen Vero cells was obtained from ECACC (Salisbury, UK). Cell samples were thawed in a $37^{\circ} \mathrm{C}$ water bath, washed by centrifugation in $10 \mathrm{ml}$ of holding medium HEPES buffered TCM 199 and suspended in Dulbecco's MEM culture medium (DMEM; Gibco BRL, Life Technologies, Paisley, Scotland) 
supplemented with $10 \%$ FCS and 1\% antibiotic-antimycotic solution, enriched with $2 \mathrm{mM}$ L-glutamine, $0.36 \mathrm{mM}$ pyruvate, and $2 \mathrm{mM}$ non-essential amino acids (Kątska-Książkiewicz et al., 2005, 2007).

Cells were seeded at the following concentrations: a. $1 \times 10^{6}$ cells in 10 $\mathrm{ml}$ medium per flask (for passages); b. $1 \times 10^{2}$ in $50 \mu 1$ medium per drop (for co-culture with the small group of embryos). The medium for co-culture $\left(\mathrm{B}_{2}\right.$ medium enriched with $10 \%$ FCS $)$ had been exchanged prior to placing of embryos and then partially exchanged every $48 \mathrm{~h}$.

\section{Assessment of caspase-3 activity in blastocysts}

Activity of caspase-3 in blastocysts was assessed using a PhiPhiLux $\mathrm{G}_{2} \mathrm{D}_{2}$ kit (Calbiochem, Darmstadt, Germany). PhiPhiLux contains a peptide substrate for caspase-3, GDEVDGI (the caspase cleavage site is underlined), which is conjugated with two rhodamine fluorophores on each site of the substrate. Upon the cleavage by active caspase- 3 high intensity red rhodamine fluorescence can be observed, while no fluorescence is emitted in intact substrate.

Embryos were placed in $50 \mu \mathrm{l}$ RPMI 1640 medium containing $10 \mu \mathrm{M}$ peptide substrate and $10 \% \mathrm{FCS}$ for $60 \mathrm{~min}$ at $38.5^{\circ} \mathrm{C}$ and $5 \% \mathrm{CO}_{2}$ in air at maximal humidity (Men et al., 2003). After incubation embryos were washed three times in PBS to reduce the background fluorescence, mounted onto a glass slide and examined under the fluorescence microscope using rhodamine filter combination (EX 510-560, BA 590).

Embryo showing red fluorescence on more than $50 \%$ of its surface was estimated as high caspase- 3 activity $(++)$. Embryo showing red fluorescence on less than $50 \%$ of its surface was estimated as low caspase-3 activity $(+)$.

\section{Experimental design}

A total of 2630 COCs (1698 COCs stained with BCB and 932 non-stained) were used for the experiments, and each experiment was replicated at least three times. We analysed the effect of COCs quality evaluated by morphology (thickness and compactness of the cumulus investment, homogeneity of the ooplasm) as well as activity of the G6PDH (evaluated by the BCB test) on the developmental potential of these oocytes after in vitro maturation and fertilization with frozenthawed semen of bulls with reduced in vitro fertilizability.

After collection of COCs, they were selected based on their morphology. COCs were classified depending on the number and compactness of the cumulus cell layers and the homogeneity of the ooplasm. Only COCs with several complete and compact cumulus cell layers and a homogeneous ooplasm were selected for experiments.

After morphological classification, the BCB test was applied. COCs $(n=1698)$ were exposed to $\mathrm{BCB}$ staining and oocytes were classified as $\mathrm{BCB}+$ or $\mathrm{BCB}-$. 
Oocytes from all groups (BCB+, $\mathrm{BCB}$ - and Control) were matured in vitro and fertilized with frozen-thawed sperm of the two bulls (P and R). To establish the relation between the quality of COCs and sperm to the quality of resulting blastocysts the activity of caspase- 3 was assessed. Blastocysts in the stages: early and middle (Day 6 to 7), late (Day 7 to 8), expanded and hatched (Day 8 to 9) were used for the assessment of caspase-3 activity.

\section{Statistical analysis}

Differences in results were assessed using a chi-square test $\left(\chi^{2}\right)$ or Fisher test (Graph-Pad software, San Diego, California, USA). Differences with a P-value of 0.05 or less were considered significant.

\section{RESULTS}

In the pool of immature, morphologically normal cumulus-oocyte-complexes recovered from cattle ovaries and stained with $\mathrm{BCB}(\mathrm{n}=1698)$, the mean percentage of COCs classified as BCB+ was $72.2 \%$ (Table 1; Figure 1).

After being classified according to COCs quality and selected by G6PDH activity using the BCB test, oocytes were subjected to IVM, and IVF, and cultured up

Table 1. Distribution of oocytes according to G6PDH activity (assessed by BCB test; blue stained oocytes $\mathrm{BCB}+$; colourless oocytes $\mathrm{BCB}-$ ) in the pool of immature, morphologically normal COCs recovered from cattle ovaries

\begin{tabular}{lcc}
\hline $\begin{array}{l}\text { No of COCs used } \\
\text { for BCB staining }\end{array}$ & $\begin{array}{c}\text { No }(\%) \text { of BCB }(+) \\
\mathrm{x} \pm \mathrm{SD}\end{array}$ & $\begin{array}{c}\text { No }(\%) \text { of BCB }(-) \\
\mathrm{x} \pm \mathrm{SD}\end{array}$ \\
\hline \multirow{2}{*}{1698} & $1226(72.2)$ & $472(27.8)$ \\
& $91.9 \pm 41.6$ & $36.4 \pm 23.6$ \\
\hline
\end{tabular}
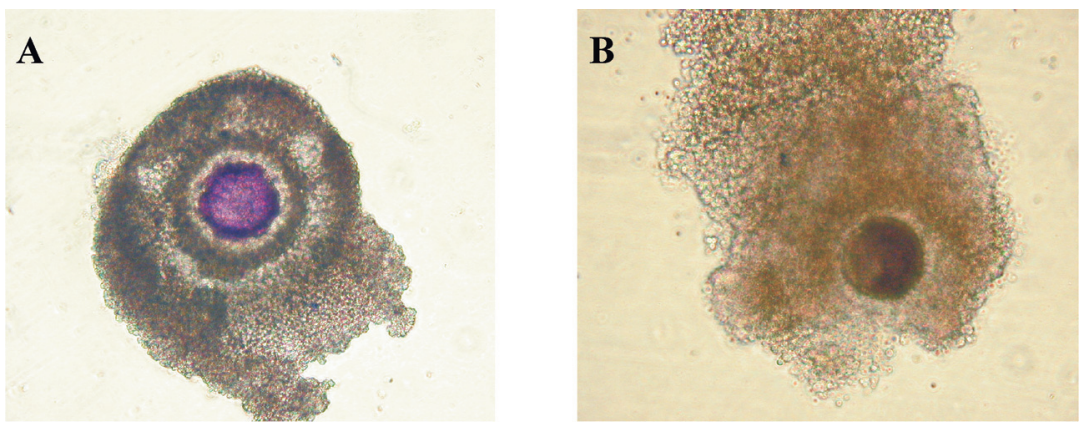

Figure 1. Immature bovine cumulus-oocyte complexes following $\mathrm{BCB}$ exposure; $\mathrm{A}-\mathrm{BCB}+$ oocyte; B - colorless BCB - oocyte $(40 \mathrm{x})$ 
to the blastocyst stage. Oocytes originating from $\mathrm{BCB}+\mathrm{COCs}$ (Figure 1) exhibited higher cleavage rates $(\mathrm{P}<0.001)$ and blastocyst rates $(\mathrm{P}<0.05)$ following IVF with sperm of bull $\mathrm{P}$ and bull $\mathrm{R}$ in comparison with BCB- oocytes (Table 2; Figure 1). A highly significant difference in cleavage rates between $\mathrm{BCB}+$ and Control oocytes was observed for the bull $\mathrm{P}(80.6$ and $70.4 \% ; \mathrm{P}<0.001)$.

Significant differences in developmental competence between $\mathrm{BCB}+$ and BCB- oocytes were observed regardless of the donor semen used (Table 2).

The highest efficiency of embryo production, regardless the semen donor, was obtained for the $\mathrm{BCB}+$ oocytes (Table 2).

Table 2. Blastocyst development from oocytes inseminated with frozen-thawed sperm of two bulls $(\mathrm{P}, \mathrm{R})$ with reduced fertilizability

\begin{tabular}{llcccc}
\hline Bull & Oocyte & $\begin{array}{c}\text { No. of } \\
\text { oocytes }\end{array}$ & $\begin{array}{c}\text { Cleavage } \\
\text { No, \% }\end{array}$ & $\begin{array}{c}\text { Blastocysts } \\
\text { No, \% }\end{array}$ & $\begin{array}{c}\text { Blastocyst/ } \\
\text { Cleaved, \% }\end{array}$ \\
\hline \multirow{2}{*}{$\mathrm{P}$} & BCB+ & 563 & $454(80.6)^{\mathrm{A}, 1}$ & $80(14.2)^{\mathrm{a}, 1}$ & $17.6^{\mathrm{a}, 1}$ \\
& BCB- & 204 & $104(51.0)^{\mathrm{B}, 1}$ & $17(8.3)^{\mathrm{b}, 1}$ & $16.3^{\mathrm{a}, 1}$ \\
& Control & 696 & $490(70.4)^{\mathrm{C}, 3}$ & $94(13.5)^{\mathrm{a}, 1}$ & $19.2^{\mathrm{a}, 1}$ \\
& BCB+ & 515 & $427(82.9)^{\mathrm{A}, 1}$ & $80(15.5)^{\mathrm{a}, 1}$ & $18.7^{\mathrm{a}, 1}$ \\
\multirow{2}{*}{$\mathrm{R}$} & BCB- & 262 & $175(66.8)^{\mathrm{B}, 2}$ & $19(7.2)^{\mathrm{b}, \mathrm{c}, 1}$ & $10.8^{\mathrm{b}, 1}$ \\
& Control & 236 & $184(78.0)^{\mathrm{A}, 1}$ & $24(10.2)^{\mathrm{b}, \mathrm{c}, 1}$ & $13.0^{\mathrm{a}, 1}$ \\
\hline
\end{tabular}

values for the same bull signed with different letters differ significantly: small letters $-\mathrm{P}<0.05$; capital letters - $\mathrm{P}<0.001$; values for the same group of oocytes signed with different Arabic numbers differ significantly: ${ }^{1,2} \mathrm{P}<0.001 ;{ }^{1: 3} \mathrm{P}<0.05$

When BCB - oocytes were used, there were significant differences in cleavage rates between $\mathrm{P}$ and $\mathrm{R}$ bulls (51.0 and 66.8\%, respectively; $\mathrm{P}<0.001$ ). However no differences between these bulls were observed in the blastocyst rates from $\mathrm{BCB}$ - oocytes (Table 2).

When control, non-stained oocytes were used for IVP, similar relationships were observed between bulls (Table 2). The significant differences $(\mathrm{P}<0.05)$ in cleavage rates were observed between bull $\mathrm{P}$ and $\mathrm{R}$ ( 70.4 and $78 \%$, respectively). However, no differences in blastocyst rate in the control oocytes were observed between bulls (Table 2).

A total number of 238 blastocysts was used for assessment of caspase-3 activity ( $84 \mathrm{BCB}+, 36 \mathrm{BCB}-$ and 118 Control) as it was presented in Table 3. A tendency towards a growth of activity of caspase-3 progressively with blastocyst development, regardless the semen used, was observed. The highest activity of caspase- 3 was assessed in the expanded and hatched blastocysts $(70.5,100$ and $58.2 \%$, respectively for $\mathrm{BCB}+, \mathrm{BCB}-$ and Control blastocysts developing after insemination with bull $\mathrm{P}$ and $70.0,80.0$ and $70.0 \%$ for bull R) (Table 3; Figure 2 ). Inverse relationships in the proportion of blastocysts with high activity of cas- 

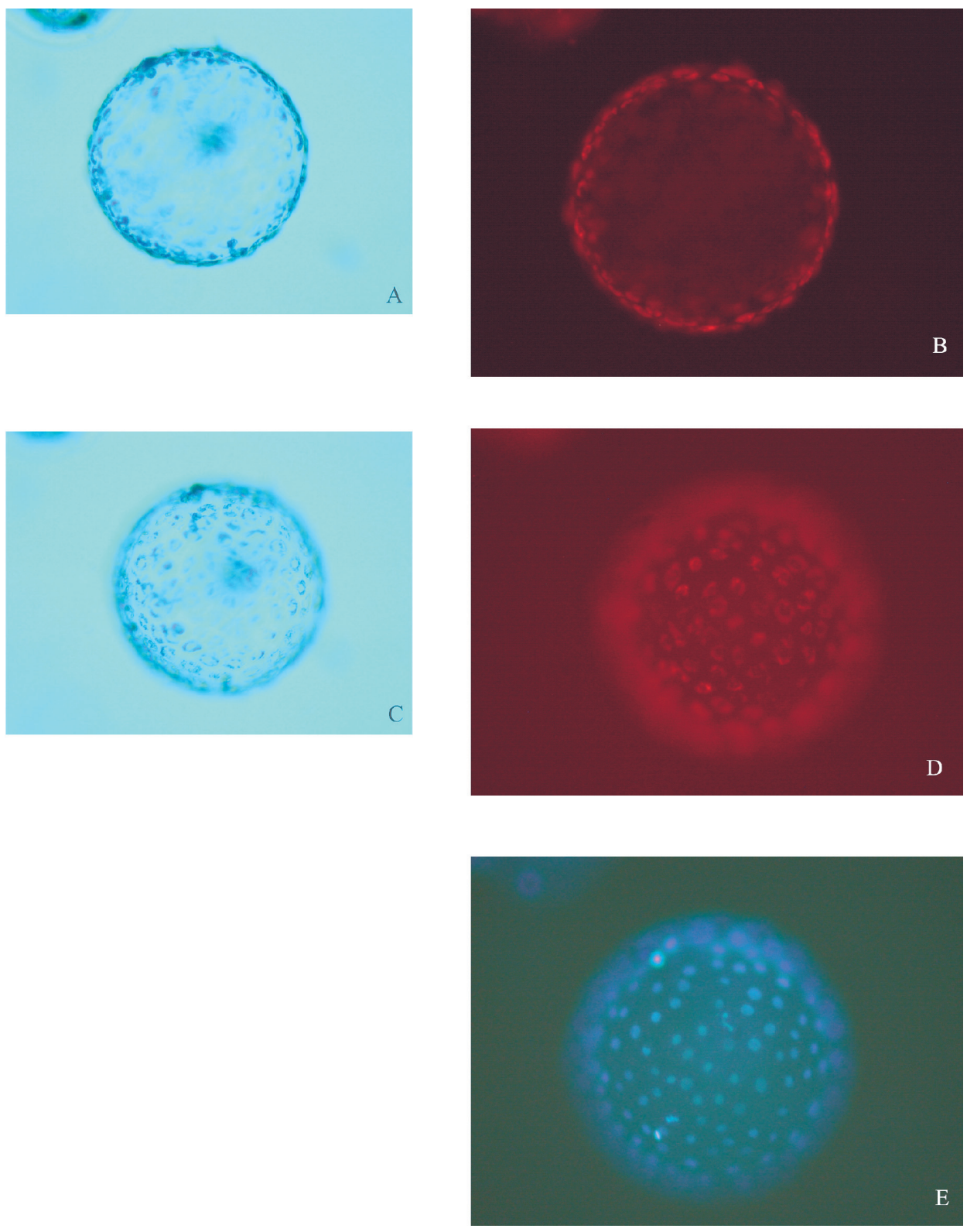

Figure 2. Caspase-3 activity in the hatched bovine blastocyst; A, C - visible light; B,D - high fluorescence (++); E - a fluorescence of nucleoli following Hoechst 33342 staining (100 x) 
Table 3. Comparison of the caspase-3 activity in blastocysts developed from oocytes inseminated with sperm of two bulls $(\mathrm{P}, \mathrm{R})$ with reduced fertilizability

\begin{tabular}{|c|c|c|c|c|c|c|c|}
\hline \multirow[b]{2}{*}{$\begin{array}{l}\text { Blastocyst } \\
\text { stage }\end{array}$} & \multirow[b]{2}{*}{$\begin{array}{l}\text { Fluorescence } \\
\text { intensity }\end{array}$} & \multicolumn{2}{|c|}{$\mathrm{BCB}+$} & \multicolumn{2}{|c|}{ BCB- } & \multicolumn{2}{|c|}{ Control } \\
\hline & & $\begin{array}{l}\text { bull P } \\
\mathrm{n}=39\end{array}$ & $\begin{array}{c}\text { bull R } \\
\mathrm{n}=45\end{array}$ & $\begin{array}{c}\text { bull P } \\
\mathrm{n}=17\end{array}$ & $\begin{array}{c}\text { bull } \mathrm{R} \\
\mathrm{n}=19\end{array}$ & $\begin{array}{l}\text { bull } \mathrm{P} \\
\mathrm{n}=94\end{array}$ & $\begin{array}{c}\text { bull R } \\
\mathrm{n}=24\end{array}$ \\
\hline \multirow{2}{*}{$\begin{array}{l}\text { Early and } \\
\text { middle }\end{array}$} & + & $5(55.5)$ & $6(60.0)$ & $4(80.0)$ & $4(66.7)$ & $12(70.6)$ & $3(50.0)$ \\
\hline & ++ & $4(44.5)$ & $4(40.0)$ & $1(20.0)$ & $2(33.3)$ & $5(29.4)$ & $3(50.0)$ \\
\hline \multirow[t]{2}{*}{ Late } & + & 7 (53.8) & $8(53.3)$ & $4(44.4)$ & $1(33.3)$ & $5(50.0)$ & $2(33.3)$ \\
\hline & ++ & $6(46.2)$ & 7 (46.7) & $5(55.6)$ & $2(66.7)$ & $5(50.0)$ & $4(66.7)$ \\
\hline \multirow{2}{*}{$\begin{array}{l}\text { Expanded } \\
\text { hatched }\end{array}$} & + & $5(29.5)$ & $6(30.0)$ & 0 & $2(20.0)$ & $28(41.8)$ & $3(30.0)$ \\
\hline & ++ & $12(70.5)$ & $14(70.0)$ & $3(100)$ & $8(80.0)$ & $39(58.2)$ & $7(70.0)$ \\
\hline
\end{tabular}

no statistical comparisons were carried out because of limited number of blastocysts at analysed stages

pase- 3 were observed for the early + middle and the late stage of blastocysts. The percentages of blastocysts with high fluorescence intensity, regardless of the origin of oocytes and sperm used, oscillated between 20 and 67\% (Table 3).

\section{DISCUSSION}

The data presented in our experiment demonstrate a relatively low rate (approximately 28\%) of oocytes with high G6PDH activity (growing or BCB- oocytes) in a pool of morphologically normal COCs recovered from ovaries of slaughtered cattle. This proportion was drastically lower compared with percentages demonstrated in a similar study by Alm et al. (2005) in which approximately $42 \%$ of COCs showed high G6PDH activity. This discrepancy seems to be understandable considering the methods of oocyte recovery used in these experiments. Namely, in the experiment of Alm et al. (2005) COCs were recovered by slicing the surface of ovaries and morphological selection of COCs was based on the compactness of cumulus investment, while in our experiment COCs were freed from ovaries following the isolation and subsequent rupture of vesicular follicles $2-8 \mathrm{~mm}$ in diameter, and additionally, during careful morphological selection of COCs prior to staining with the $\mathrm{BCB}$, we excluded most COCs with small oocytes.

It was hypothesized that the additional selection of COCs by BCB staining, would allow the selection of fully grown, and therefore the most competent oocytes from a highly heterogeneous pool originating from ovarian follicles at different stages of growth and atresia. As a consequence, careful, double oocyte selection should improve embryo production efficiency. It was shown in the present study that the developmental competence of $\mathrm{BCB}+$ (low G6PDH activity) and Control oocytes was significantly higher in comparison with BCB- (high G6PDH 
activity) oocytes. This implies that the BCB test is a useful tool for selection of more developmentally competent oocytes. These results are in agreement with similar studies in cattle (Pujol et al., 2004; Alm et al., 2005; Bhojwani et al., 2007), goat (Rodriguez-González et al., 2003; Kątska-Książkiewicz et al., 2007) and pig (Wongsrikeao et al., 2006). Selection of excellent quality oocytes is highly desirable for procedures involving extensive manipulations such as pronuclear microinjections, intracytoplasmic sperm injection, and nuclear transplantation, which requires high developmental competence after embryo transfer. Due to the specificity of the methods mentioned above, the BCB test might be a preferable selection method. Therefore, it may be assumed that accurate, careful selection of COCs recovered from ovaries of adult cattle provides reasonable and sufficient means for oocyte quality analysis and suitability for IVM/IVF procedures. However, even a homogenous population of oocytes with similar cumulus cell mass cannot guarantee the same developmental competence. Thus, the female effect on IVP results was also considered (Lonergan et al., 2003; Palma and Sinowatz, 2004). It seems that several replications of IVP with reasonable amounts of oocytes allows the elimination, at least to some degree, of vastly ranging developmental competence of oocytes.

It is well known that the outcome of IVP can be affected by several factors. Besides the already discussed oocyte quality effect, a second important factor affecting IVP success rate depends on the sperm. Several authors working on in vitro embryo production in cattle described variability among bulls in the ability of their sperm to become capacitated, fertilize oocytes and subsequently produce competent embryos that develop to the blastocyst stage (Chamberland et al., 2001; Vassena et al., 2003; Palma and Sinowatz, 2004; Kątska-Książkiewicz et al., 1996, 2005, 2006). In terms of factors affecting the outcome of IVF, bull variability has been the main source of difficulty in this type of work. These bull-to-bull variations in the outcome of in vitro embryo production frequently lead to selection of bulls used for IVF. Also, in several other mammalian species, male-to-male variations in the outcome of in vitro embryo production have been reported (Cognie, 1999; Kątska-Książkiewicz et al., 2007). For these reasons semen from a male of proven fertility is commonly used for IVF purposes.

As it was already mentioned, both semen samples used in the present experiment were to some extent uniform with respect to motility, quality of chromatin, survival time and capacity of freezing. However, in the previous IVP trials these bulls have been evaluated as less suitable to IVF. It would be reasonable to include for the experiment additionally a control bull producing sperm of accepted in vitro fertilizability. The lack of data produced by a control bull of a proven fertility is a problem in this experiment. We all know that each IVP system has its intrinsic effect on embryo quality, therefore by showing the data we consider as satisfactory it would be far more easier to convince the reader that the observed 
phenomenon is due to the bull effect and not to the culture system. Therefore, due to limited number of oocytes obtained per trial, this issue had to be omitted. However, it seemed interesting to compare IVP outcomes using carefully selected oocytes with frozen-thawed sperm of these negatively selected bulls. Indeed, the hypothesis that sperm produced by such bulls may perform better in vitro when using oocytes selected by the BCB staining appeared to be true. Nevertheless, in comparison to results of our previous experiments (Kątska-Książkiewicz et al., 1996, 2005) and several other reports (Blondin et al., 2002; Lonergan et al., 2003; Machatkova et al., 2004; Alm et al., 2005; Hasler, 2006; Sirard et al., 2006) the overall blastocyst rates using semen of the two negatively selected bulls still remained relatively low.

In the present work we used the caspase- 3 activity as a quality marker of blastocysts produced from oocytes with different G6PDH activity. Indeed, the blastocyst quality produced in our experiment could be additionally characterized by the number of cells and hatching ability. However, considering that the blastocysts for analysis were gradually pick-up from culture (Days: 6-7, 7-8, 8-9), the calculation of hatching rates could not be assessed. Moreover, to avoid additional manipulations with blastocysts used for analysis of caspase-3 activity we decided not determine number of cells in all analysed blastocysts.

A tendency towards growth of activity of caspase-3 with blastocyst development was observed. The highest activity of caspase- 3 was assessed in the expanded and hatched blastocysts. It has been demonstrated by Gjǿrret et al. (2003, 2007 ) that appearance of apoptotic characteristics is accelerated by in vitro conditions but their number is insignificant prior to compaction of embryo. The dead cell index (DCI, total number of apoptotic nuclei/total number of nuclei) tends to increase as the in vitro culture time increases (Neuber et al., 2002). In our experiment a significant overbalance of blastocysts with high caspase-3 activity, regardless the stage of blastocyst development and their origin, was noticed. We assumed that not only in vitro conditions but also lowered fertilizability sperm might induce the incidence of apoptosis in resulting embryos. However, due to the shortage of blastocysts, especially in the BCB- group, the statistical analysis couldn't be done. Recently Gjǿrret et al. (2007) analysed caspase-3 activity and ultrastructural evidence of apoptosis in bovine in vitro produced embryos. According to these authors apoptotic features were presented in all analysed bovine blastocysts.

\section{CONCLUSIONS}

Summarizing the results of our experiment, we demonstrated that both, the quality of cumulus-oocyte-complexes and sperm influence the efficiency of in 
vitro embryo production in cattle. The highest blastocyst rates have been observed for brilliant cresyl blue + oocytes regardless the source of semen. Therefore, using excellent quality oocytes and sperm with reduced in vitro fertilizability it was possible to overcome (to some extent) the bull effect on efficiency of in vitro fertilization. However, the overall efficiency of blastocysts production using semen of the two negatively selected bulls still remained relatively low.

\section{REFERENCES}

Aguilar M.M., Galina C.S., Merchant H., Montiel F., Canseco R., Márquez Y.C., 2002. Comparison of stereoscopy, light microscopy and ultrastructural methods for evaluation of bovine embryos. Reprod. Domest. Anim. 37, 341-346

Alm H., Torner H., Löhrke B., Viergutz T., Ghoneim I.M., Kanitz W., 2005. Bovine blastocyst development rate in vitro is influenced by selection of oocytes by brilliant cresyl blue staining before IVM as indicator for glucose-6-phosphate dehydrogenase activity. Theriogenology 63, 2194-2205

Bhojwani S., Alm H., Torner H., Kanitz W., Poehland R., 2007. Selection of developmentally competent oocytes through brilliant cresyl blue stain enhances blastocyst development rate after bovine nuclear transfer. Theriogenology 67, 341-345

Blondin P., Bosquet D., Twagiramungu H., Barnes F., Sirard M.A., 2002. Manipulation of follicular development to produce developmentally competent oocytes. Biol. Reprod. 66, 38-43

Bols P.E.J., Goovaerts I.G.F., Leroy J.L.M.R., 2007. From retrieval to blastocyst culture: following the individual oocyte. In: Proceedings of $23^{\text {rd }}$ Scientific Meeting of European Embryo Transfer Association (AETE). Alghero (Sardinia), pp.79-89

Chamberland A., Fournier V., Tardif S., Sirard M.A., Sullivan R., Bailey J.L., 2001. The effect of heparin on motility parameters and protein phosphorylation during bovine sperm capacitation. Theriogenology 55, 823-835

Cognie Y., 1999. State of the art in sheep-goat embryo transfer. Theriogenology 51, 105-116

Gjørret J.O., Fabian D., Avery B., Maddox-Hyttel P., 2007. Active caspase-3 and ultrastructural evidence of apoptosis in spontaneous and induced cell death in bovine in vitro produced preimplantation embryos. Mol. Reprod. Dev. 74, 961-971

Gjørret J.O., Knijn H.M., Dieleman S.J., Avery B., Larsson L.I., Maddox-Hyttel P., 2003. Chronology of apoptosis in bovine embryos produced in vivo and in vitro. Biol. Reprod. 69,1193-1200

Han Z.B., Lan G.C., Wu Y.G., Han D., Feng W.G., Wang J.Z., Tan J.H., 2006. Interactive effects of granulosa cell apoptosis, follicle size, cumulus-oocyte complex morphology, and cumulus expansion on the developmental competence of goat oocytes: a study using the well-in-drop culture system. Reproduction 132, 749-758

Hasler J.F., 2006. In vitro production of cattle embryos. In: Proceedings of $22^{\text {nd }}$ Scientific Meeting of the European Society of Embryo Transfer (AETE). Zug (Switzerland), pp. 95-104

Kątska-Książkiewicz L., Bochenek M., Ryńska B., 2005. Effect of quality of sperm chromatin structure on in-vitro production of cattle embryos. Arch. Tierzucht 48, 32-39

Kątska-Książkiewicz L., Opiela J., Ryńska B., 2007. Effects of oocyte quality, semen donor and embryo co-culture system on the efficiency of blastocyst production in goats. Theriogenology 68, 736-744

Kątska-Książkiewicz L., Ryńska B., Bochenek M., Opiela J., Jurkiewicz J., 2006. In vitro production of bovine embryos using flow-cytometrically sexed sperm. Arch. Tierzucht 49, 133-140 
Kątska L., Ryńska B., Smorag Z., 1996. Effect of seminal plasma on the in vitro fertilizability of bull spermatozoa. Anim. Reprod. Sci. 44, 23-31

Lonergan P., Rizos D., Gutierrez-Adan A., Fair T., Boland M.P., 2003. Oocyte and embryo quality: effect of origin, culture conditions and gene expression patterns. Reprod. Domest. Anim. 38, 259-267

Machatkova M., Krausova K., Jokesova E., Tomanek M., 2004. Developmental competence of bovine oocytes: effects of follicle size and the phase of follicular wave on in vitro embryo production. Theriogenology 61, 329-335

Men H., Monson R.L., Parrish J.J., Rutledge J.J., 2003. Degeneration of cryopreserved bovine oocytes via apoptosis during subsequent culture. Cryobiology 47, 73-81

Neuber E., Luetjens C.M., Chan A.W., Schatten G.P., 2002. Analysis of DNA fragmentation of in vitro cultured bovine blastocysts using TUNEL. Theriogenology 57, 2193-2202

Palma G.A., Sinowatz F., 2004. Male and female effects on the in vitro production of bovine embryos. Anat. Histol. Embryol. 33, 257-262

Pavlok A., Čech S., Kubelka M., Lopatáŕová M., Holy L., Jindra M., 2006. Storage of bovine isolated follicles: A new alternative way to improve the recovery rate of viable embryos from ovarian follicles of slaughtered cows. Anim. Reprod. Sci. 96, 186-195

Pujol M., Lopez-Béjar M., Paramio M.T., 2004. Developmental competence of heifer oocytes selected using the brilliant cresyl blue (BCB) test. Theriogenology 61, 735-744

Rizos D., Ward F., Duffy P., Boland M.P., Lonergan P., 2002. Consequences of bovine oocyte maturation, fertilization or early embryo development in vitro versus in vivo: implications for blastocyst yield and blastocyst quality. Mol. Reprod. Dev. 61, 234-248

Rodriguez-González E., López-Béjar M., Izquierdo D., Paramio M.T., 2003. Developmental competence of prepubertal goat oocytes selected with brilliant cresyl blue and matured with cysteamine supplementation. Reprod. Nutr. Develop. 43, 179-187

Sirard M.A., Richard F., Blondin P., Robert C., 2006. Contribution of the oocyte to embryo quality. Theriogenology 65,126-136

Tian W.N., Braunstein L.D., Pang J., Stuhlmeier K.M., Xi Q.C., Tian X., Stanton R.C., 1998. Importance of glucose-6-phosphate dehydrogenase activity for cell growth. J. Biol. Chem. 273,10609-10617

Vassena R., Mapletoft R.J., Allodi S., Singh J., Adams G.P., 2003. Morphology and developmental competence of bovine oocytes relative to follicular status. Theriogenology 60, 923-932

Wongsrikeao P., Otoi T., Yamasaki H., Agung B., Taniguchi M., Naoi H., Shimizu R., Nagai T., 2006. Effects of single and double exposure to brilliant cresyl blue on the selection of porcine oocytes for in vitro production of embryos. Theriogenology $66,366-372$ 